\title{
芳基吡啶酮腙类化合物的设计、合成及杀菌活性研究
}

\author{
王家尧陶晗金蜜李丽莎 \\ 肖玉梅李佳奇覃兆海* \\ (中国农业大学理学院 北京 100193)
}

\begin{abstract}
摘要 以吡啶二芳酮为分子插件, 以嘧菌腙为母体化合物设计并合成了一系列新型芳基吡啶酮腙类化合物. 产物结构 经 ${ }^{1} \mathrm{H} N M R 、{ }^{13} \mathrm{C} \mathrm{NMR}$ 及 HRMS 确认, 并采用生长速率法对所有化合物进行了离体抑菌活性测试. 结果表明: 在浓度 为 $70 \mu \mathrm{mol} / \mathrm{L}$ 时, 大部分化合物对所选 8 种病菌具有一定的抑制活性, 其中 2'-甲基乙酰基苯-4,6-二甲氧基嘧啶-2-腙 (III-3)和 2-(2'-((4"-溴-苯基)(3"-氯-吡啶-4"-基)-亚甲基)-肼基)-4,6-二甲氧基嘧啶(III-18)对病原菌抑制效果明显高于对 照药嘧菌腙. III-3 对番茄灰霉病菌 $\mathrm{EC}_{50}$ 为 $22.18 \mu \mathrm{mol} / \mathrm{L}$ (嘧菌腙为 $31.38 \mu \mathrm{mol} / \mathrm{L}$ ), III-18 对水稻纹枯病菌 $\mathrm{EC}_{50}$ 小于 0.35 $\mu \mathrm{mol} / \mathrm{L}$, 比对照药嘧菌腙提高了 260 倍以上.
\end{abstract}

关键词 分子插件; 吡啶二芳酮; 芳基吡啶酮腙; 抑菌活性

\section{Design, Synthesis and Activity Evaluation of Arylpyridone Hydrazones}

\author{
Wang, Jiayao Tao, Han Jin, Mi Li, Lisha \\ Xiao, Yumei Li, Jiaqi Qin, Zhaohai* \\ (College of Science, China Agricultural University, Beijing 100193)
}

\begin{abstract}
A series of novel arylpyridone hydrazones were designed and synthesized by using arylpyridinylmethanone as the plug-in molecules and ferimzone as parent compound. The structure of the products were confirmed by ${ }^{1} \mathrm{H}$ NMR, ${ }^{13} \mathrm{C}$ NMR and HRMS. The antifungal activities of all compounds were tested by growth rate method in vitro. The results showed that most of the compounds inhibited eight selected pathogens at the concentration of $70 \mu \mathrm{mol} / \mathrm{L}$. The inhibitory effect of 2'-methylacetylbenzene-4,6-dimethoxypyrimidine-2-hydrazone (III-3) and 2-(2'-((4'-bromo-phenyl)(3'-chloro-pyridine-4'-yl)methylene-hydrazine)-4,6-dimethoxypyrimidine (III-18) on pathogenic fungi was significantly higher than that of ferimzone, in which compound III-3 has a great degree of inhibition on Botrytis cinerea with $\mathrm{EC}_{50}$ value of $22.18 \mu \mathrm{mol} / \mathrm{L}$, and $\mathrm{EC}_{50}$ value of compound III-18 is less than $0.35 \mathrm{umol} / \mathrm{L}$ on Thanatephorus cucumeris, which is 260 times higher than that of control.
\end{abstract}

Keywords plug-in molecule; arylpyridinylmethanone; arylpyridone hydrazone; antifungal activity

1,1-二芳基化合物在医药和农药产品中都有着广泛 而重要的应用和较高的出现频率 ${ }^{[1 \sim 3]}$, 如在农药上, 鼠 特灵(Norbormide)用作灭鼠剂 ${ }^{[4,5]}$, 充当血管收缩剂和钲 通道阻滞剂, 对大鼠有选择性毒性, 而对其他物种毒性 较低; 日本石原公司开发的 Pyriofenone ${ }^{[6]}$ 对谷类、葡萄 和蔬菜粉霉病有非常好的杀菌活性, 尤其对灰霉病有特 效; 在医药上, 抗心律失常的药物磷酸丙吡胺 (Disopyramide $)^{[7]}$ 和吡美诺 (Pimenol) $)^{[8]}$ 、脂肪酸酰胺水解 酶(FAAH) 抑制剂 VER-24052 ${ }^{[9]}$ 和抗组胺药氯苯吡醇胺

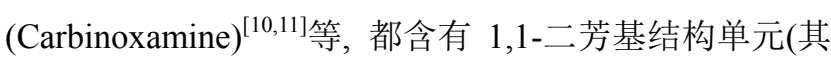
中一个芳基为吡啶基)(图 1). 可见 1,1-二芳基结构是一 类重要的结构单元.

因此，基于 1,1-二芳基结构的广泛应用以及我们提 出的 “分子插件” (The Plug-in Molecules) ${ }^{[12]}$ 的概念, 并 结合吡啶杂环在农药分子中的独特作用, 我们建立了吡 啶二芳酮(1)分子插件数据库, 并成功应用于新农药开 发中. 在前期工作中, 我们以该类分子插件为基础, 合 成了多个系列的新型活性化合物(图 2), 如通过 Wittig-

* Corresponding author. E-mail: qinzhaohai@263.net

Received October 16, 2018; revised December 5, 2018; published online December 28, 2018.

Project supported by the National Natural Science Foundation of China (No. 21572265).

国家自然科学基金(No. 21572265)资助项目. 
<smiles>O=C1NC(=O)C2C1C1C=CC2C23C=CC2C(=C(c2ccccc2)c2ccccn2)C13</smiles>

Norbormide<smiles>COc1cc(C)c(C(=O)c2c(OC)ncc(Cl)c2C)c(OC)c1OC</smiles>

Pyriofenone<smiles>CC(C)CN(C)CCC(C(N)=O)(c1ccccc1)c1ccccn1</smiles>

Disopyramide<smiles>CC1CCCC(C)N1CCCC(O)(c1ccccc1)c1ccccn1</smiles>

Pimenol<smiles>O=C(N1CCCCC1)N1CC(OC(c2ccc(Cl)cc2)c2ncccc2Cl)C1</smiles>

VER-2452<smiles>CN(C)CCOC(c1ccc(Cl)cc1)c1ccccn1</smiles>

Carbinoxamine

图 1 代表性 1-芳基-1-吡啶基医药和农药品种

Figure 1 Representative 1-aryl-1-pyridine based medicine and pesticide varieties

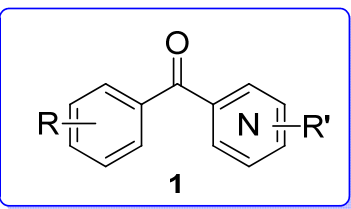<smiles>[R]C=CC(=NOC(=O)c1c(Oc2nc(OC)cc(OC)n2)cccc1Oc1nc(OC)cc(OC)n1)C(=NOC)c1ccccc1C(=NOC)C(=O)OC</smiles>

图 2 吡啶二芳酮类分子插件及其应用

Figure 2 Aplication examples of arylpyridinylmethanone plug-in molecules

Horner 反应合成了二芳基丙烯酰胺类化合物, 开发出了 新型杀菌剂丁吡吗啉 $(2)^{[13 \sim 16]}$, 它既能影响病原菌细胞 壁合成物质的极性分布, 又能通过抑制病原菌细胞呼吸 链复合物 III 的活性而抑制其能量合成, 现已商品化投 入市场应用; 通过 Knoevenagel 反应合成了水合丁吡吗 啉(3)及其类似物 ${ }^{[17]}$, 其中化合物 3 的活性与丁吡吗啉相 当; 以其肜为原料合成的甲氧丙烯酸酯类化合物 4 对小 麦白粉病、黄瓜炭疽病具有很好的防效, 并对稻梨孢的 孢子萌发具有强烈的抑制效果 ${ }^{[18]}$; 以其肜与双草醚形 成的肟酯 5 对稗草的除草活性优于嘧啶肜草醚 ${ }^{[19]}$ 等. 可 见吡啶二芳酮类是一类功能强大的分子插件.

嘧菌腙(Ferimzone, 6) 是由日本武田公司开发的一 种农用杀菌剂, 主要防治水稻上由稻尾声孢、稻长蠕孢 和稻梨狍等病原菌引起的病害. 其能干扰真菌细胞膜的 通透性, 从而影响离子和小分子的流通 ${ }^{[20]}$. 本研究以嘧
菌腙为母体化合物，设计并合成了一系列芳基吡啶酮腙 类化合物 III (Eq. 1), 将吡啶杂环、嘧啶杂环和腙单元有 效整合到同一分子中，测定了它们的杀菌活性，并对其 构效关系进行了初步探讨.

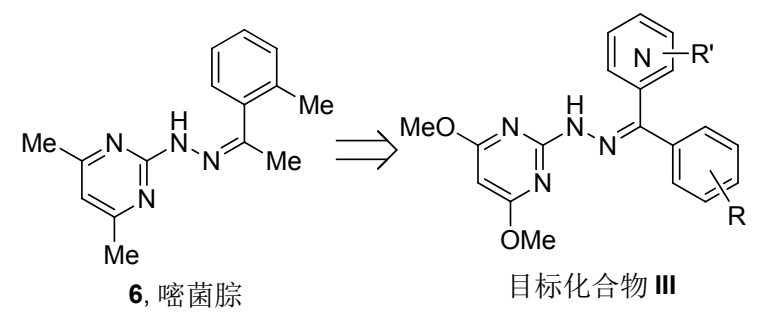

\section{1 结果与讨论}

\section{1 目标化合物的合成}

目标化合物的合成见 Scheme 1 所示，以 4,6-二甲氧 
基-2-甲磺酰基嘧啶为起始原料, 首先通过肼解 ${ }^{[21]}$ 得到 4,6-二甲氧基-2-肼基嘧啶(II), 此步反应通过抽滤可直 接得到白色固体产物, 收率 $85 \%$. 然后用化合物 II 与各 类芳酮进行亲核加成消除反应得到腙 ${ }^{[22]}$ III-1 III-26. 大部分化合物可通过重结晶直接得到, 少数化合物通过 柱层析后结晶获得. 化合物结构及反应收率如表 1 所示.

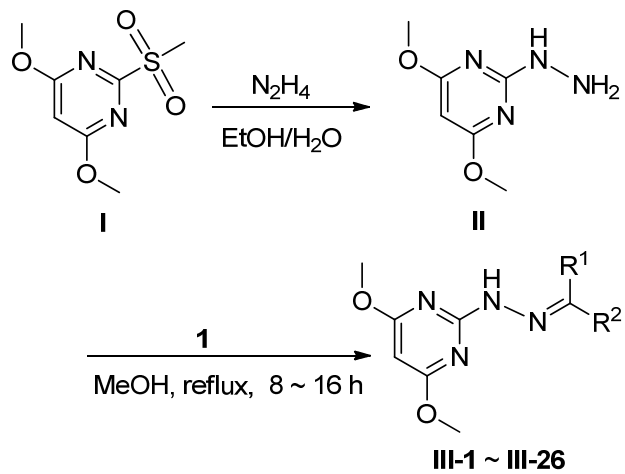

图式 1 目标化合物的合成

Scheme 1 Synthesis of target compounds

表 1 目标化合物结构及收率

Table 1 Structure and yield of target compounds

\begin{tabular}{|c|c|c|c|}
\hline Compd. & $\mathrm{R}^{1}$ & $\mathrm{R}^{2}$ & Yield $/ \%$ \\
\hline III-1 & $\mathrm{Ph}$ & $\mathrm{Ph}$ & 75 \\
\hline III-2 & 4- ${ }^{t} \mathrm{BuC}_{6} \mathrm{H}_{4}$ & $4-\mathrm{NO}_{2} \mathrm{C}_{6} \mathrm{H}_{4}$ & 82 \\
\hline III-3 & $\mathrm{CH}_{3}$ & $2-\mathrm{CH}_{3} \mathrm{C}_{6} \mathrm{H}_{4}$ & 67 \\
\hline III-4 & $4-{ }^{t} \mathrm{BuC}_{6} \mathrm{H}_{4}$ & 3-Pyridyl & 88 \\
\hline III-5 & $3,4-\mathrm{Cl}_{2} \mathrm{C}_{6} \mathrm{H}_{3}$ & 3-Pyridyl & 62 \\
\hline III-6 & $4-\mathrm{CH}_{3} \mathrm{C}_{6} \mathrm{H}_{4}$ & 4-Pyridyl & 90 \\
\hline III-7 & 4- ${ }^{i} \mathrm{PrC}_{6} \mathrm{H}_{4}$ & 4-Pyridyl & 68 \\
\hline III-8 & $3,4-\left(\mathrm{CH}_{3}\right)_{2} \mathrm{C}_{6} \mathrm{H}_{3}$ & 4-Pyridyl & 65 \\
\hline III-9 & $2-\mathrm{CH}_{3}-3-\mathrm{ClC}_{6} \mathrm{H}_{3}$ & 4-Pyridyl & 70 \\
\hline III-10 & $4-\mathrm{BrC}_{6} \mathrm{H}_{4}$ & 4-Pyridyl & 61 \\
\hline III-11 & $4-\mathrm{BrC}_{6} \mathrm{H}_{4}$ & 2-Cl-3-pyridyl & 63 \\
\hline III-12 & $4-{ }^{t} \mathrm{BuC}_{6} \mathrm{H}_{4}$ & 2-Cl-3-pyridyl & 56 \\
\hline III-13 & $4-\mathrm{CH}_{3} \mathrm{C}_{6} \mathrm{H}_{4}$ & 2-Cl-3-pyridyl & 55 \\
\hline III-14 & $3,4-\mathrm{Cl}_{2} \mathrm{C}_{6} \mathrm{H}_{3}$ & 2-Cl-3-pyridyl & 64 \\
\hline III-15 & $2,4-\left(\mathrm{CH}_{3}\right)_{2} \mathrm{C}_{6} \mathrm{H}_{3}$ & 3-Cl-4-pyridyl & 91 \\
\hline III-16 & $4-\mathrm{CH}_{3} \mathrm{C}_{6} \mathrm{H}_{4}$ & 3-Cl-4-pyridyl & 93 \\
\hline III-17 & $3,4-\left(\mathrm{CH}_{3}\right)_{2} \mathrm{C}_{6} \mathrm{H}_{3}$ & 3-Cl-4-pyridyl & 78 \\
\hline III-18 & $4-\mathrm{BrC}_{6} \mathrm{H}_{4}$ & 3-Cl-4-pyridyl & 72 \\
\hline III-19 & 4- $\mathrm{PhC}_{6} \mathrm{H}_{4}$ & 3-Cl-4-pyridyl & 91 \\
\hline III-20 & $3-\mathrm{NO}_{2}-4-\mathrm{CH}_{3} \mathrm{C}_{6} \mathrm{H}_{3}$ & 3-Cl-4-pyridyl & 68 \\
\hline III-21 & $4-{ }^{t} \mathrm{BuC}_{6} \mathrm{H}_{4}$ & 3-Cl-4-pyridyl & 88 \\
\hline III-22 & $4-\mathrm{CH}_{3} \mathrm{C}_{6} \mathrm{H}_{4}$ & 4-Cl-3-pyridyl & 64 \\
\hline III-23 & $2,4-\left(\mathrm{CH}_{3}\right)_{2} \mathrm{C}_{6} \mathrm{H}_{3}$ & 4-Cl-3-pyridyl & 65 \\
\hline III-24 & 4- $\mathrm{BrC}_{6} \mathrm{H}_{4}$ & 4-Cl-3-pyridyl & 89 \\
\hline III-25 & 4- $\left(4^{\prime}-\mathrm{Br}-\mathrm{C}_{6} \mathrm{H}_{4}\right) \mathrm{C}_{6} \mathrm{H}_{4}$ & 4-Cl-3-pyridyl & 66 \\
\hline III-26 & $3,5-\mathrm{Cl}_{2} \mathrm{C}_{6} \mathrm{H}_{3}$ & 4-Cl-3-pyridyl & 61 \\
\hline
\end{tabular}

1.2 抑菌活性初篮测定

通过菌丝生长速率法 ${ }^{[23]}$ 测试了目标化合物在 0.07 $\mathrm{mmol} / \mathrm{L}$ 浓度下对棉花立枯(Rhizoctonia Cotton, $\mathrm{RC}$ )、水
稻稻瘟(Pyricularia oryzae Cav, PC)、番茄灰霉(Botrytis cinerea, $\mathrm{BC})$ 、番茄晚疫(Phytophthora infestans, PI)、水 稻纹枯 (Thanatephorus cucumeris, TC)、小麦赤霉 (FusaHum graminearum Sehw, FS)、油菜菌核(Sclerotinia sclerotiorum, SS)和玉米大斑(Exserohilum turcicum, ET) 八种病原菌的抑制效果, 结果列于表 2 .

由表 2 抑制率数据可知: 在 $0.07 \mathrm{mmol} / \mathrm{L}$ 浓度下, 对 于棉花立枯病菌、水稻稻瘟病菌来说，除化合物 III-4 对 棉花立枯病菌抑制活性优于对照药剂嘧菌腙外，其他化 合物表现出较差的抑制活性. 对于番茄灰霉病菌来说, 化合物 III-3 和 III-11 表现出优秀的抑制活性，抑制率分 别达到 $84.86 \%$ 和 $74.68 \%$, 有进一步改造的潜力. 对于 番茄晚疫病菌来说，约 7/9 的化合物抑制活性高于对照 药剂嘧菌腙, 其中化合物 III-3, III-11 和 III-14 抑制率接 近嘧菌腙的 2 倍. 对于水稻纹枯病菌, 化合物 III-18 明 显优于其他 25 个化合物, 且比对照药剂嘧菌腙抑制率 高 $17 \%$. 对于小麦赤霉病菌, 相比较基本没有药效的嘧 菌腙, 所设计的大多数化合物有中等程度的药效, 可见 吡啶二芳酮基团的引入对于防治小麦赤霉病菌起到很 好的作用，这对后续防治小麦赤霉病菌的药剂开发有借 鉴意义. 对于油菜菌核病菌, 化合物 III-3 抑制活性最 佳, 且高于嘧菌腙, 其他化合物抑制活性表现较差. 对 于玉米大斑病菌，化合物 III-3 和 III-22 抑制活性好于对 照药剂, 化合物 III-1 和 III-11 也有和对照药剂相当的活 性. 总的来说, 所设计合成的 26 个化合物均表现出一定 的抑制活性，体现出化合物设计的合理性，同时化合物 III-3, III-11, III-14 和 III-18 整体表现出较好的抑制活性, 有进一步研究的价值.

化合物的构效关系: (1)当 $\mathrm{R}^{1}$ 基团为 $4-\mathrm{CH}_{3}-\mathrm{C}_{6} \mathrm{H}_{4}$ 时, $\mathrm{R}^{2}$ 基团分别为 4-Pyridyl (III-6), 2-Cl-3-Pyridyl (III-13), 3-Cl-4-Pyridyl (III-16)和 4-Cl-3-Pyridyl (III-22), 通过比 较其抑制活性可以看出, 对于番茄灰霉病菌、油菜菌核 病菌和稻瘟病菌, 化合物 III-13 和 III-22 活性明显高于 另外两个化合物, 对于其他 6 种病原菌, 这 4 个化合物 没有表现出明显的规律性，可见 $\mathrm{R}^{2}$ 基团为 3-Pyridyl 有 一定优势. (2)当 $\mathrm{R}^{1}$ 基团为 4- $\mathrm{Br}-\mathrm{C}_{6} \mathrm{H}_{4}$ 时, $\mathrm{R}^{2}$ 基团分别为 4-Py (III-10), 2-Cl-3-Pyridyl (III-11), 3-Cl-4-Pyridyl (III-18)和 4-Cl-3-Pyridyl (III-24), 比较 III-11 和 III-24 的抑制活性数据，可知对于篮选的所有 8 种病原菌，化 合物 III-11 抑制活性均明显优于化合物 III-24, 可见 $\mathrm{R}^{2}$ 基团 3-吡啶环上氯原子位于 2-位的抑制活性优于 4-位. 比较 III-10 和 III-18 的抑制活性数据则没有发现明显规 律性. (3)当 $\mathrm{R}^{1}$ 基团为 $3,4-\left(\mathrm{CH}_{3}\right)_{2} \mathrm{C}_{6} \mathrm{H}_{3}$ 时, $\mathrm{R}^{2}$ 基团分别为 4-Pyridyl (III-8)和 3-Cl-4-Pyridyl (III-13)时, 对于除油 菜菌核病菌外的 7 种病原菌，化合物 III-8 抑制活性均明 
显优于化合物 III-13，可见 $\mathrm{R}^{2}$ 基团 4-吡啶环上 3 -位引入 氯原子不利于提高其抑制活性. (4) 当 $R^{2}$ 基团为 4-Pyridyl, $\mathrm{R}^{1}$ 基团分别为 $4-\mathrm{CH}_{3} \mathrm{C}_{6} \mathrm{H}_{4}$ (III-6), $4-{ }^{i} \mathrm{PrC}_{6} \mathrm{H}_{4}$ (III-7), 3,4- $\left(\mathrm{CH}_{3}\right)_{2} \mathrm{C}_{6} \mathrm{H}_{3}$ (III-8) 和 2- $\mathrm{CH}_{3}-3-\mathrm{ClC}_{6} \mathrm{H}_{3}$ (III-9), 通过分析其抑制活性数据可以发现, 多取代的 III-8 和 III-9 对于 8 种病原菌的抑制效果明显好于单取代的化 合物 III-6 和 III-7. 同时当 $\mathrm{R}^{2}$ 基团为 3-Cl-4-Pyridyl 时, $\mathrm{R}^{1}$ 基团分别为 $3,4-\left(\mathrm{CH}_{3}\right)_{2} \mathrm{C}_{6} \mathrm{H}_{3}$ (III-17)和 $4-\mathrm{CH}_{3} \mathrm{C}_{6} \mathrm{H}_{4}$ (III-16), 分析其生测数据, 发现对除棉花立枯病菌外的 7 种病原菌, 多取代的 III-17 明显比单取代 III-16 抑制 效果好，但当 $\mathrm{R}^{1}$ 基团为 $2,4-\left(\mathrm{CH}_{3}\right)_{2} \mathrm{C}_{6} \mathrm{H}_{3}$ (III-15)时，活性 相比 III-17 下降很明显, 说明多取代的位置对活性影响 也十分关键.

\section{3 精密毒力的测定}

经初步实验篮选, 选取活性较好的化合物 III-3, III-11, III-14 和 III-18 进行油菜菌核(Sclerotinia sclerotiorum)、水稻稻瘟(Pyricularia oryzae Cav)、番茄晚疫 (Phytophthora infestans)、番茄灰霉(Botrytis cinerea) 和水 稻纹枯(Thanatephorus cucumeris)五种病原菌的精密毒
力实验，实验采用生长速率法测定 $140,70,35,3.5,0.35$ 和 $0 \mu \mathrm{mol} \cdot \mathrm{L}^{-1}$ 浓度下的抑制率, 实验均重复三次, $\mathrm{EC}_{50}$ 通过社会科学统计程序(SPSS)计算, 结果列于表 3 .

通过表 3 数据可以看出, 所有生测的相关系数均大 于 0.8 , 可见浓度与抑制率数据具有很强的相关性. 对 于油菜菌核病菌, 化合物 III-3 的 $\mathrm{EC}_{50}$ 比嘧菌腙降低了 一半, 同时化合物 III-3 对番茄晚疫病菌和番茄灰霉病 菌上的药效是嘧菌腙的 1.5 倍以上. 化合物 III-11 和 III-14 在番茄晚疫病菌上的 $\mathrm{EC}_{50}$ 值比嘧菌腙明显要低. 有趣的是, 化合物 III-18 在水稻纹枯病菌的 $\mathrm{EC}_{50}$ 值比嘧 菌腙要低 260 倍以上, 可见其在很低的浓度就能起到很 好的抑制效果，通过其毒力回归方程可以发现，化合物 III-18 斜率很低, 说明该化合物即使浓度变化很大，其 药效影响变化并不大.

\section{2 结论}

通过分子插件原理，设计并合成了 26 个芳基吡啶 酮腙类化合物，除 III-1 是已知化合物外，共有 25 个新 化合物. 测试了棉花立枯、水稻稻瘟、番茄灰霉、番茄

表 2 目标化合物 III-1 III-26 的抑菌活性(抑制率/\% $)^{a}$

Table 2 Antifungal activity of title compounds III-1 $\sim$ III-26 (inhibitory rate/\%)

\begin{tabular}{|c|c|c|c|c|c|c|c|c|}
\hline Compd. & $\mathrm{RC}$ & $\mathrm{PC}$ & $\mathrm{BC}$ & PI & $\mathrm{TC}$ & FS & SS & ET \\
\hline 嘧菌腙 & 32.76 & 55.19 & 69.74 & 34.62 & 52.31 & 8.48 & 98.30 & 90.80 \\
\hline III-1 & 19.38 & 51.75 & 42.92 & 62.50 & 25.46 & 38.45 & 52.45 & 89.68 \\
\hline III-2 & 0 & 2.26 & 8.80 & 4.43 & 0 & 19.98 & 11.34 & 46.17 \\
\hline III-3 & 20.29 & 42.75 & 84.86 & 65.23 & 41.48 & 33.06 & 100 & 91.66 \\
\hline III-4 & 43.51 & 14.33 & 24.68 & 45.51 & 32.41 & 30.61 & 8.30 & 0 \\
\hline III-5 & 0 & 26.69 & 38.82 & 61.01 & 39.41 & 44.61 & 33.22 & 57.97 \\
\hline III-6 & 17.94 & 25.41 & 32.19 & 40.17 & 29.40 & 35.92 & 25.76 & 81.49 \\
\hline III-7 & 20.62 & 17.77 & 35.62 & 36.97 & 13.89 & 26.23 & 10.97 & 84.52 \\
\hline III-8 & 31.13 & 37.96 & 57.51 & 57.69 & 42.13 & 46.29 & 27.95 & 84.07 \\
\hline III-9 & 29.69 & 41.41 & 66.09 & 55.98 & 43.98 & 49.05 & 25.76 & 91.25 \\
\hline III-10 & 28.04 & 27.13 & 61.80 & 55.98 & 26.39 & 37.76 & 43.96 & 84.19 \\
\hline III-11 & 25.77 & 46.82 & 74.68 & 65.17 & 38.89 & 37.07 & 74.28 & 89.01 \\
\hline III-12 & 20.21 & 39.44 & 66.09 & 53.63 & 29.63 & 42.14 & 60.94 & 80.71 \\
\hline III-13 & 0 & 38.46 & 51.69 & 40.74 & 42.17 & 40.23 & 68.60 & 68.40 \\
\hline III-14 & 1.48 & 42.53 & 47.62 & 66.08 & 27.19 & 34.48 & 65.89 & 75.93 \\
\hline III-15 & 3.09 & 3.00 & 6.87 & 0.21 & 6.02 & 5.49 & 11.45 & 1.30 \\
\hline III-16 & 17.53 & 5.71 & 33.48 & 50.85 & 8.33 & 8.71 & 32.31 & 5.79 \\
\hline III-17 & 0 & 14.70 & 51.01 & 44.33 & 13.83 & 29.83 & 53.20 & 20.52 \\
\hline III-18 & 0 & 17.64 & 43.79 & 50.46 & 68.91 & 30.10 & 50.77 & 22.57 \\
\hline III-19 & 0 & 9.05 & 15.57 & 11.82 & 8.30 & 15.87 & 17.28 & 86.87 \\
\hline III-20 & 0 & 7.46 & 32.05 & 46.66 & 6.91 & 28.19 & 36.46 & 75.24 \\
\hline III-21 & 0 & 2.71 & 20.31 & 6.54 & 1.15 & 17.51 & 27.55 & 20.52 \\
\hline III-22 & 16.91 & 26.88 & 57.08 & 47.01 & 19.91 & 27.39 & 53.42 & 91.48 \\
\hline III-23 & 0 & 38.91 & 45.37 & 57.63 & 16.36 & 26.54 & 48.34 & 47.88 \\
\hline III-24 & 0 & 12.67 & 36.11 & 52.99 & 18.90 & 32.56 & 52.39 & 0 \\
\hline III-25 & 0 & 0 & 7.00 & 6.76 & 0 & 1.37 & 43.75 & 86.19 \\
\hline III-26 & 0 & 22.85 & 34.31 & 34.20 & 13.83 & 29.28 & 45.10 & 62.07 \\
\hline
\end{tabular}


表 3 部分化合物的 $\mathrm{EC}_{50}$

Table $3 \quad \mathrm{EC}_{50}$ of part title compounds

\begin{tabular}{|c|c|c|c|c|}
\hline 病原菌 & 化合物 & $\mathrm{EC}_{50} /\left(\mu \mathrm{mol} \cdot \mathrm{L}^{-1}\right)$ & $\mathrm{R}^{2}$ & 毒力回归方程 \\
\hline \multirow{5}{*}{ SS } & 嘧菌腙 & $52.29 \pm 1.54$ & 0.999 & $y=1.19 x-0.78$ \\
\hline & III-3 & $36.39 \pm 6.68$ & 0.938 & $y=2.13 x-2.77$ \\
\hline & III-11 & $60.27 \pm 5.74$ & 0.979 & $y=1.62 x-2.9$ \\
\hline & III-14 & $67.96 \pm 5.81$ & 0.992 & $y=0.78 x-1.43$ \\
\hline & III-18 & $>140$ & & \\
\hline \multirow{5}{*}{$\mathrm{PC}$} & 嘧菌腙 & $48.11 \pm 4.99$ & 0.940 & $y=1.19 x-2.05$ \\
\hline & III-3 & $99.04 \pm 5.64$ & 0.999 & $y=1.09 x-2.18$ \\
\hline & III-11 & $110.97 \pm 5.01$ & 0.984 & $y=1.24 x-2.53$ \\
\hline & III-14 & $>140$ & & \\
\hline & III-18 & $>140$ & & \\
\hline \multirow{5}{*}{ PI } & 嘧菌腙 & $96.99 \pm 9.06$ & 0.999 & $y=2.44 x-4.85$ \\
\hline & III-3 & $55.96 \pm 7.57$ & 0.992 & $y=2.04 x-3.53$ \\
\hline & III-11 & $74.71 \pm 3.77$ & 0.970 & $y=0.92 x-1.73$ \\
\hline & III-14 & $61.33 \pm 2.00$ & 0.935 & $y=0.53 x-0.96$ \\
\hline & III-18 & $99.81 \pm 10.74$ & 0.971 & $y=0.75 x-1.5$ \\
\hline \multirow{5}{*}{$\mathrm{BC}$} & 嘧菌腙 & $31.38 \pm 1.83$ & 0.821 & $y=1.53 x-2.26$ \\
\hline & III-3 & $22.18 \pm 6.03$ & 0.953 & $y=1.51 x-1.96$ \\
\hline & III-11 & $>140$ & & \\
\hline & III-14 & $>140$ & & \\
\hline & III-18 & $>140$ & & \\
\hline \multirow{5}{*}{$\mathrm{TC}$} & 嘧菌腙 & $93.33 \pm 9.41$ & 0.998 & $y=2.2 x-4.33$ \\
\hline & III-3 & $>140$ & & \\
\hline & III-11 & $>140$ & & \\
\hline & III-14 & $>140$ & & \\
\hline & III-18 & $<0.35$ & 0.998 & $y=0.11 x+0.18$ \\
\hline
\end{tabular}

晚疫、水稻纹枯、小麦赤霉、油菜菌核和玉米大斑八种 病原菌的抑制效果, 发现大多数化合物都有一定的抑制 活性，可见分子插件原理可以实现化合物的快速合成与 筷选. 同时化合物 III-3 和 III-18 对病原菌抑制效果明显 高于对照药嘧菌腙, III-11, III-14 和其他部分化合物也 有不弱于嘧菌腙的效果, 本研究对新型杀菌剂的设计合 成提供有益的参考, 具有重要的借鉴意义.

\section{3 实验部分}

\section{1 仪器与试剂}

Bruker Avance DPX300 超导核磁共振仪(TMS 为内 标); 安捷伦 ESI-Q-TOF 型高分辨质谱仪; Yanagimoto MFG Co. 熔点测定仪, 温度计未经校正; 宁波江南仪器 厂 RXZ 智能型光照培养箱; 所有试剂国产市售分析纯, 无水溶剂按标准方法处理.

\section{2 实验方法}

\section{2 .1 嘧菌腙(6)的合成}

$100 \mathrm{~mL}$ 三口瓶中加入 4.6-二甲基-2-氯嘧啶 $(2 \mathrm{~g}$, $14.1 \mathrm{mmol}$ )、磁子和 $40 \mathrm{~mL}$ 乙醇, 于室温搅拌. 然后加入 水合肼(0.92 mL, $15.5 \mathrm{mmol})$. 加热回流 $8 \mathrm{~h}$, 冷却抽滤得
淡黄色固体 4.6-二甲基-2-肼基嘧啶 $1.75 \mathrm{~g}$, 收率 90\%.

取 $1 \mathrm{~g}$ 4.6-二甲基-2-肼基嘧啶(7.2 $\mathrm{mmol}$ )加入 100 $\mathrm{mL}$ 圆底烧瓶, 加入邻甲基苯乙酮 $(0.92 \mathrm{~g}, 6.9 \mathrm{mmol}) 、$ 磁 子和 $40 \mathrm{~mL}$ 无水甲醇. 常温搅拌 $10 \mathrm{~min}$ 后加入 1 滴乙 酸, 加热回流 $8 \mathrm{~h}$. 冷却抽滤得淡黄色固体 $1.7 \mathrm{~g}$. 收率 93\%. m.p. $182.4 \sim 183.6{ }^{\circ} \mathrm{C}$ (文献值 ${ }^{[24]}$ : m.p. $178 \sim$ $\left.180{ }^{\circ} \mathrm{C}\right) ;{ }^{1} \mathrm{H}$ NMR $\left(300 \mathrm{MHz}, \mathrm{CDCl}_{3}\right) \delta: 7.76(\mathrm{~s}, 1 \mathrm{H})$, $7.30 \sim 7.14(\mathrm{~m}, 3 \mathrm{H}), 7.04 \sim 6.98(\mathrm{~m}, 1 \mathrm{H}), 6.39(\mathrm{~s}, 1 \mathrm{H})$, $2.28(\mathrm{~s}, 6 \mathrm{H}), 2.26(\mathrm{~s}, 3 \mathrm{H}), 2.18(\mathrm{~s}, 3 \mathrm{H}) ;{ }^{13} \mathrm{C} \mathrm{NMR}(75$ $\left.\mathrm{MHz}, \mathrm{CDCl}_{3}\right) \delta: 167.70,159.25,149.54,134.46,130.61$, $128.89,126.56,126.40,111.94,24.71,23.59,18.62$; HRMS (ESI) calcd for $\mathrm{C}_{15} \mathrm{H}_{19} \mathrm{~N}_{4}(\mathrm{M}+\mathrm{H})^{+} 255.1604$ found 255.1599 .

\subsection{2 目标化合物 III-1 III-26 的合成}

$250 \mathrm{~mL}$ 三口瓶中加入 4.6-二甲氧基-2-甲磺酰基嘧 啶 $(20 \mathrm{~g}, 91.7 \mathrm{mmol})$ 和磁子, 加入 $120 \mathrm{~mL}$ 乙醇于室温搅 拌. 然后加入水合肼 $(5.8 \mathrm{~mL}, 100.9 \mathrm{mmol})$, 加热回流 8 h. 冷却抽滤得白色固体 $13.3 \mathrm{~g}$, 收率 $85 \%$.

取 4.6-二甲氧基-2-肼基基嘧啶 $(0.5 \mathrm{~g}, 2.9 \mathrm{mmol}$ )加 入 $100 \mathrm{~mL}$ 圆底烧瓶, 加入二苯甲酮 $(0.51 \mathrm{~g}, 2.8 \mathrm{mmol}) 、$ 磁子和 $40 \mathrm{~mL}$ 无水甲醇. 常温搅拌 $10 \mathrm{~min}$ 后加入 1 滴乙 酸, 加热回流 $8 \mathrm{~h}$, 薄层色谱(TLC)监测反应完毕. 柱层 析分离得白色固体 III-1. 用同样的方法得到目标化合 物 III-2 III-26.

苯甲酰基苯-4,6-二甲氧基嘧啶-2-腙(III-1)：收率 $75 \%$. m.p. $158.1 \sim 159.2{ }^{\circ} \mathrm{C} ;{ }^{1} \mathrm{H}$ NMR $(300 \mathrm{MHz}$, DMSO-d $\left.d_{6}\right) \delta: 8.19(\mathrm{~s}, 1 \mathrm{H}), 7.68 \sim 7.61(\mathrm{~m}, 2 \mathrm{H}), 7.59 \sim$ $7.47(\mathrm{~m}, 3 \mathrm{H}), 7.35 \sim 7.28(\mathrm{~m}, 5 \mathrm{H}), 5.57(\mathrm{~s}, 1 \mathrm{H}), 3.91(\mathrm{~s}$, $6 \mathrm{H}) ;{ }^{13} \mathrm{C} \mathrm{NMR}\left(75 \mathrm{MHz}, \mathrm{CDCl}_{3}\right) \delta: 172.10,158.55$, $148.44,137.35,132.01,129.41,129.22,128.60,128.40$, $127.84,126.82,81.15,53.58$.

2-[2'-(4"-叔丁基-苯基)(4"-硝基-苯基)-亚甲基]-肼 基)-4,6-二甲氧基嘧啶(III-2)：收率 82\%. m.p. 213.4 $214.9{ }^{\circ} \mathrm{C}$; ${ }^{1} \mathrm{H}$ NMR (300 MHz, $\left.\mathrm{CDCl}_{3}\right) \delta: 8.41(\mathrm{~s}, 1 \mathrm{H})$, $8.19 \sim 8.12(\mathrm{~m}, 2 \mathrm{H}), 7.82 \sim 7.76(\mathrm{~m}, 2 \mathrm{H}), 7.64 \sim 7.59(\mathrm{~m}$, $2 \mathrm{H}), 7.26 \sim 7.21(\mathrm{~m}, 2 \mathrm{H}), 5.62(\mathrm{~s}, 1 \mathrm{H}), 3.93(\mathrm{~s}, 6 \mathrm{H}), 1.40$ $(\mathrm{s}, 9 \mathrm{H}) ;{ }^{13} \mathrm{C} \mathrm{NMR}\left(75 \mathrm{MHz}, \mathrm{CDCl}_{3}\right) \delta: 172.22,158.19$, $153.05,147.26,146.02,143.66,128.03,127.55,127.26$, 126.71, 123.12, 53.77, 34.65, 30.90; HRMS (ESI) calcd for $\mathrm{C}_{23} \mathrm{H}_{26} \mathrm{~N}_{5} \mathrm{O}_{4}(\mathrm{M}+\mathrm{H})^{+} 436.1979$, found 436.1980.

2'-甲基乙酰基苯-4,6-二甲氧基嘧啶-2-腙(III-3): 收 率 67\%. m.p. 150.6 151.2 ${ }^{\circ} \mathrm{C}$; ${ }^{1} \mathrm{H}$ NMR (300 MHz, $\left.\mathrm{CDCl}_{3}\right) \delta: 8.12(\mathrm{~s}, 1 \mathrm{H}), 7.39 \sim 7.32(\mathrm{~m}, 1 \mathrm{H}), 7.22 \sim 7.18$ $(\mathrm{m}, 3 \mathrm{H}), 5.59(\mathrm{~s}, 1 \mathrm{H}), 3.92(\mathrm{~s}, 6 \mathrm{H}), 2.52(\mathrm{~s}, 3 \mathrm{H}), 2.28(\mathrm{~s}$, $3 \mathrm{H}) ;{ }^{13} \mathrm{C}$ NMR $\left(75 \mathrm{MHz}, \mathrm{CDCl}_{3}\right) \delta: 172.05,159.12$, 
147.92, 139.07, 135.79, 130.70, 127.97, 127.71, 125.29, 81.29, 53.57, 20.87, 15.82; HRMS (ESI) calcd for $\mathrm{C}_{15} \mathrm{H}_{19} \mathrm{~N}_{4} \mathrm{O}_{2}(\mathrm{M}+\mathrm{H})^{+}$287.1503, found 287.1499.

2-(2'-((4"-叔丁基-苯基)(吡啶-3"-基)-亚甲基)-肼 基)-4,6-二甲氧基嘧啶(III-4)：收率 88\%. m.p. 180.6 $181.9{ }^{\circ} \mathrm{C} ;{ }^{1} \mathrm{H}$ NMR $\left(300 \mathrm{MHz}, \mathrm{CDCl}_{3}\right) \delta: 8.52(\mathrm{dd}, J=4.6$, $1.6 \mathrm{~Hz}, 2 \mathrm{H}), 8.40$ (s, 1H), 7.57 (d, $J=8.5 \mathrm{~Hz}, 2 \mathrm{H}), 7.47$ (dd, $J=4.6,1.6 \mathrm{~Hz}, 2 \mathrm{H}), 7.21(\mathrm{~d}, J=8.5 \mathrm{~Hz}, 2 \mathrm{H}), 5.58$ (s, $1 \mathrm{H}), 3.90(\mathrm{~s}, 6 \mathrm{H}), 1.36(\mathrm{~s}, 9 \mathrm{H}) ;{ }^{13} \mathrm{C}$ NMR $(75 \mathrm{MHz}$, $\left.\mathrm{CDCl}_{3}\right) \delta: 172.14,158.21,152.84,149.46,145.64,144.72$, 128.03, 127.31, 126.53, 120.60, 81.55, 53.64, 34.57, 30.87; HRMS (ESI) calcd for $\mathrm{C}_{22} \mathrm{H}_{26} \mathrm{~N}_{5} \mathrm{O}_{2}(\mathrm{M}+\mathrm{H})^{+} 392.2081$, found 392.2078 .

2-(2'-((3,4"-二氯-苯基)(吡啶-3"-基)-亚甲基)-肼 基)-4,6-二甲氧基嘧啶(III-5)：收率 62\%. m.p. 189.8 $190.9{ }^{\circ} \mathrm{C} ;{ }^{1} \mathrm{H}$ NMR $\left(300 \mathrm{MHz}, \mathrm{CDCl}_{3}\right) \delta: 8.74$ (d, $J=2.2$ $\mathrm{Hz}, 1 \mathrm{H}), 8.51$ (dd, $J=4.8,1.6 \mathrm{~Hz}, 1 \mathrm{H}), 8.24$ (s, 1H), 7.91 (d, $J=8.1 \mathrm{~Hz}, 1 \mathrm{H}), 7.70(\mathrm{~d}, J=8.3 \mathrm{~Hz}, 2 \mathrm{H}), 7.28 \sim 7.15$ (m, 3H), 5.58 (s, 1H), 3.88 (s, 6H); ${ }^{13} \mathrm{C}$ NMR $(75 \mathrm{MHz}$, $\left.\mathrm{CDCl}_{3}\right) \delta: 172.06,158.24,149.40,148.12,144.13,133.46$, $133.01,132.78,130.02,129.62,124.09,122.76,81.87$, 53.65; HRMS (ESI) calcd for $\mathrm{C}_{18} \mathrm{H}_{16} \mathrm{C}_{12} \mathrm{~N}_{5} \mathrm{O}_{2}(\mathrm{M}+\mathrm{H})^{+}$ 404.0676, found 404.0675.

2-(2'-((4"- 甲基-苯基)(吡啶 -4"- 基)-亚甲基)-肼 基)-4,6-二甲氧基嘧啶(III-6): 收率 90\%. m.p. 169.8 $170.5{ }^{\circ} \mathrm{C} ;{ }^{1} \mathrm{H}$ NMR $\left(300 \mathrm{MHz}, \mathrm{CDCl}_{3}\right) \delta: 8.55(\mathrm{dd}, J=4.7$, $1.6 \mathrm{~Hz}, 2 \mathrm{H}), 8.39$ (s, 1H), 7.49 (dd, $J=4.7,1.6 \mathrm{~Hz}, 2 \mathrm{H})$, $7.39(\mathrm{~d}, J=8.1 \mathrm{~Hz}, 2 \mathrm{H}), 7.19$ (d, $J=8.0 \mathrm{~Hz}, 2 \mathrm{H}), 5.61(\mathrm{~s}$, $1 \mathrm{H}), 3.92(\mathrm{~s}, 6 \mathrm{H}), 2.46(\mathrm{~s}, 3 \mathrm{H}) ;{ }^{13} \mathrm{C}$ NMR $(75 \mathrm{MHz}$, $\left.\mathrm{CDCl}_{3}\right) \delta: 172.11,158.19,149.50,145.69,144.70,139.79$, $130.31,128.20,127.40,120.59,81.80,53.63,21.10$; HRMS (ESI) calcd for $\mathrm{C}_{19} \mathrm{H}_{20} \mathrm{~N}_{5} \mathrm{O}_{2}(\mathrm{M}+\mathrm{H})^{+} 350.1612$, found 350.1613 .

2-(2'-((4"-异丙基-苯基)(吡啶-4"-基)-亚甲基)-肼 基)-4,6-二甲氧基嘧啶(III-7)：收率 68\%. m.p. 173.5 $174{ }^{\circ} \mathrm{C} ;{ }^{1} \mathrm{H}$ NMR $\left(300 \mathrm{MHz}, \mathrm{CDCl}_{3}\right) \delta: 8.56$ (dd, $J=4.6$, $1.6 \mathrm{~Hz}, 2 \mathrm{H}), 8.43(\mathrm{~s}, 1 \mathrm{H}), 7.51(\mathrm{dd}, J=4.6,1.6 \mathrm{~Hz}, 2 \mathrm{H})$, $7.45(\mathrm{~d}, J=8.1 \mathrm{~Hz}, 2 \mathrm{H}), 7.24(\mathrm{~d}, J=8.1 \mathrm{~Hz}, 2 \mathrm{H}), 5.62(\mathrm{~s}$, $1 \mathrm{H}), 3.93(\mathrm{~s}, 6 \mathrm{H}), 3.01(\mathrm{dt}, J=13.8,6.9 \mathrm{~Hz}, 1 \mathrm{H}), 1.34(\mathrm{~d}$, $J=6.9 \mathrm{~Hz}, 6 \mathrm{H}) ;{ }^{13} \mathrm{C}$ NMR $\left(75 \mathrm{MHz}, \mathrm{CDCl}_{3}\right) \delta: 172.11$, $158.20,150.51,149.47,145.65,144.71,128.27,127.68$, $120.58,81.62,53.61,33.71,23.44$; HRMS (ESI) calcd for $\mathrm{C}_{21} \mathrm{H}_{24} \mathrm{~N}_{5} \mathrm{O}_{2}(\mathrm{M}+\mathrm{H})^{+}$378.1925, found 378.1922.

2-(2'-((3,4"-二甲基-苯基)(吡啶-4"-基)-亚甲基)-肼 基)-4,6-二甲氧基嘧啶(III-8)：收率 65\%. m.p. 191.2
$192{ }^{\circ} \mathrm{C} ;{ }^{1} \mathrm{H}$ NMR $\left(300 \mathrm{MHz}, \mathrm{CDCl}_{3}\right) \delta: 8.62 \sim 8.48(\mathrm{~m}$, $3 \mathrm{H}), 7.51(\mathrm{dd}, J=4.6,1.6 \mathrm{~Hz}, 2 \mathrm{H}), 7.33 \sim 7.25(\mathrm{~m}, 1 \mathrm{H})$, $7.02(\mathrm{~d}, J=7.0 \mathrm{~Hz}, 2 \mathrm{H}), 5.60$ (s, 1H), 3.91 (s, 6H), 2.32 (d, $J=4.4 \mathrm{~Hz}, 6 \mathrm{H}) ;{ }^{13} \mathrm{C}$ NMR $\left(75 \mathrm{MHz}, \mathrm{CDCl}_{3}\right) \delta: 172.00$, $158.21,149.44,145.84,144.77,138.35,138.12,130.67$, 129.00, 127.88, 125.56, 120.57, 81.74, 53.53, 19.50, 19.34; HRMS (ESI) calcd for $\mathrm{C}_{20} \mathrm{H}_{22} \mathrm{~N}_{5} \mathrm{O}_{2}(\mathrm{M}+\mathrm{H})^{+} 364.1768$, found 364.1769 .

2-(2'-((2"-甲基-3"-氯-苯基)(吡啶-4"-基)-亚甲基) 肼基)-4,6-二甲氧基嘧啶(III-9)：收率 70\%. m.p. 152.0 $152.8{ }^{\circ} \mathrm{C} ;{ }^{1} \mathrm{H}$ NMR $\left(300 \mathrm{MHz}, \mathrm{CDCl}_{3}\right) \delta: 8.59(\mathrm{~s}, 1 \mathrm{H})$, 8.47 (dd, $J=4.6,1.6 \mathrm{~Hz}, 2 \mathrm{H}), 7.46 \sim 7.37$ (m, 3H), $7.09 \sim$ $6.96(\mathrm{~m}, 2 \mathrm{H}), 5.53(\mathrm{~d}, J=0.9 \mathrm{~Hz}, 1 \mathrm{H}), 3.81(\mathrm{~s}, 6 \mathrm{H}), 2.35$ $(\mathrm{d}, J=3.3 \mathrm{~Hz}, 3 \mathrm{H}) ;{ }^{13} \mathrm{C} \mathrm{NMR}\left(75 \mathrm{MHz}, \mathrm{CDCl}_{3}\right) \delta: 171.88$, $158.07,149.46,144.26,144.21,144.18,143.72,137.90$, $137.86,135.89,135.67,132.03,130.49,130.25,129.58$, $129.07,128.63,126.92,126.45,120.32,82.17,53.52$, 19.83, 19.66; HRMS (ESI) calcd for $\mathrm{C}_{19} \mathrm{H}_{19} \mathrm{ClN}_{5} \mathrm{O}_{2}$ (M+ $\mathrm{H})^{+}$384.1222, found 384.1219 .

2-(2'-((4"-溴-苯基)(吡啶-4"-基)-亚甲基)-肼基)-4,6二甲氧基嘧啶(III-10): 收率 $61 \%$. m.p. $180.8 \sim 182{ }^{\circ} \mathrm{C}$; ${ }^{1} \mathrm{H}$ NMR $\left(300 \mathrm{MHz}, \mathrm{CDCl}_{3}\right) \delta: 8.85(\mathrm{dd}, J=4.3,1.6 \mathrm{~Hz}$, 2H), $7.98(\mathrm{~s}, 1 \mathrm{H}), 7.43(\mathrm{~s}, 4 \mathrm{H}), 7.25(\mathrm{dd}, J=4.3,1.6 \mathrm{~Hz}$, $2 \mathrm{H}), 5.59(\mathrm{~s}, 1 \mathrm{H}), 3.89(\mathrm{~s}, 6 \mathrm{H}) ;{ }^{13} \mathrm{C}$ NMR $(75 \mathrm{MHz}$, $\left.\mathrm{CDCl}_{3}\right) \delta 172.09,158.17,151.24,143.94,139.65,135.00$, 131.25, 127.92, 123.33, 123.08, 81.92, 53.66; HRMS (ESI) calcd for $\mathrm{C}_{18} \mathrm{H}_{17} \mathrm{BrN}_{5} \mathrm{O}_{2}(\mathrm{M}+\mathrm{H})^{+} 414.0560$, found 414.0564 .

2-(2'-((4"-溴-苯基)(2"-氯-吡啶-3"-基)-亚甲基)-肼 基)-4,6-二甲氧基嘧啶(III-11)：收率 63\%. m.p. 190 $190.9{ }^{\circ} \mathrm{C} ;{ }^{1} \mathrm{H}$ NMR $\left(300 \mathrm{MHz}, \mathrm{CDCl}_{3}\right) \delta: 8.60$ (dd, $J=4.8$, $2.0 \mathrm{~Hz}, 1 \mathrm{H}), 7.91(\mathrm{~s}, 1 \mathrm{H}), 7.66(\mathrm{dd}, J=7.5,2.0 \mathrm{~Hz}, 1 \mathrm{H})$, $7.50 \sim 7.39(\mathrm{~m}, 5 \mathrm{H}), 5.61(\mathrm{~s}, 1 \mathrm{H}), 3.91(\mathrm{~s}, 6 \mathrm{H}) ;{ }^{13} \mathrm{C} \mathrm{NMR}$ $\left(75 \mathrm{MHz}, \mathrm{CDCl}_{3}\right) \delta: 172.08,158.10,151.12,150.13$, $141.77,139.77,134.67,131.37,127.53,127.10,123.28$, $123.09,81.95,53.78$; HRMS (ESI) calcd for $\mathrm{C}_{18} \mathrm{H}_{16} \mathrm{Br}-$ $\mathrm{ClN}_{5} \mathrm{O}_{2}(\mathrm{M}+\mathrm{H})^{+} 448.0170$, found 448.0166 .

2 - $(2$ '-((4"-叔丁基-苯基)(2"-氯-吡啶-3"-基)-亚甲 基)-肼基)-4,6-二甲氧基嘧啶(III-12): 收率 56\%. m.p. $179.1 \sim 179.3{ }^{\circ} \mathrm{C} ;{ }^{1} \mathrm{H}$ NMR $\left(300 \mathrm{MHz}, \mathrm{CDCl}_{3}\right) \delta: 8.54(\mathrm{dd}$, $J=4.8,2.0 \mathrm{~Hz}, 1 \mathrm{H}), 7.85(\mathrm{~s}, 1 \mathrm{H}), 7.60(\mathrm{dd}, J=7.5,2.0 \mathrm{~Hz}$, $1 \mathrm{H}), 7.44 \sim 7.33(\mathrm{~m}, 5 \mathrm{H}), 5.55(\mathrm{~s}, 1 \mathrm{H}), 3.85(\mathrm{~s}, 6 \mathrm{H}) .1 .33$ $(\mathrm{s}, 9 \mathrm{H}) ;{ }^{13} \mathrm{C}$ NMR $\left(75 \mathrm{MHz}, \mathrm{CDCl}_{3}\right) \delta: 172.13,158.16$, $151.11,150.16,141.71,139.78,134.68,131.37,127.53$, 127.13, 123.27, 123.08, 81.96, 53.75. 34.65, 30.88; HRMS 
(ESI) calcd for $\mathrm{C}_{22} \mathrm{H}_{25} \mathrm{ClN}_{5} \mathrm{O}_{2}(\mathrm{M}+\mathrm{H})^{+}$426.1691, found 426.1688 .

2-(2'-((4"-甲基-苯基)(2"-氯-吡啶-3"-基)-亚甲基)肼基)-4,6-二甲氧基嘧啶 (III-13): 收率 55\%. m.p. $173.9 \sim 174.4{ }^{\circ} \mathrm{C} ;{ }^{1} \mathrm{H}$ NMR $\left(300 \mathrm{MHz}, \mathrm{CDCl}_{3}\right) \delta: 8.59$ (dd, $J=4.8,2.0 \mathrm{~Hz}, 1 \mathrm{H}), 7.89$ (s, 1H), 7.66 (dd, $J=7.5,2.0 \mathrm{~Hz}$, $1 \mathrm{H}), 7.50 \sim 7.41(\mathrm{~m}, 3 \mathrm{H}), 7.14(\mathrm{~d}, J=8.1 \mathrm{~Hz}, 2 \mathrm{H}), 5.59$ (s, $1 \mathrm{H}), 3.91(\mathrm{~s}, 6 \mathrm{H}), 2.34(\mathrm{~s}, 3 \mathrm{H}) ;{ }^{13} \mathrm{C} \mathrm{NMR}(75 \mathrm{MHz}$, $\left.\mathrm{CDCl}_{3}\right) \delta: 172.07,158.32,150.82,150.10,143.13,139.78$, $139.16,132.94,128.92,127.85,126.08,122.98,81.63$, 53.70, 20.99; HRMS (ESI) calcd for $\mathrm{C}_{19} \mathrm{H}_{19} \mathrm{ClN}_{5} \mathrm{O}_{2}(\mathrm{M}+$ $\mathrm{H})^{+}$384.1222, found 384.1224.

2-(2'-((3,4"-二氯-苯基)(2"-氯一吡啶-3"- 基 $)$ - 亚甲 基)-肼基)-4,6-二甲氧基嘧啶(III-14)：收率 64\%. m.p. 178.2 $179{ }^{\circ} \mathrm{C} ;{ }^{1} \mathrm{H}$ NMR (300 MHz, $\left.\mathrm{CDCl}_{3}\right) \delta: 8.61$ (dd, $J=4.8,2.0 \mathrm{~Hz}, 1 \mathrm{H}), 7.95(\mathrm{~s}, 1 \mathrm{H}), 7.70 \sim 7.61(\mathrm{~m}, 2 \mathrm{H})$, $7.52 \sim 7.48(\mathrm{~m}, 1 \mathrm{H}), 7.40 \sim 7.32(\mathrm{~m}, 2 \mathrm{H}), 5.62(\mathrm{~s}, 1 \mathrm{H})$, $3.90(\mathrm{~s}, 6 \mathrm{H}) ;{ }^{13} \mathrm{C} \mathrm{NMR}\left(75 \mathrm{MHz}, \mathrm{CDCl}_{3}\right) \delta: 172.13$, $158.02,151.32,150.11,140.31,139.78,135.82,132.87$, $132.62,130.15,127.62,126.62,125.12,123.17,82.19$, 53.78; HRMS (ESI) calcd for $\mathrm{C}_{18} \mathrm{H}_{15} \mathrm{C}_{13} \mathrm{~N}_{5} \mathrm{O}_{2}(\mathrm{M}+\mathrm{H})^{+}$ 438.0286, found 438.0285 .

2-(2'-((2,4"-二甲基-苯基)(3"-氯一吡啶-4"-基)-亚甲 基)-肼基)-4,6-二甲氧基嘧啶(III-15)：收率 91\%. m.p. 207.8 208 ${ }^{\circ} \mathrm{C} ;{ }^{1} \mathrm{H}$ NMR (300 MHz, $\left.\mathrm{CDCl}_{3}\right) \delta: 8.28(\mathrm{~d}$, $J=5.3 \mathrm{~Hz}, 1 \mathrm{H}), 8.17$ (s, 1H), 7.44 (dd, $J=5.3,1.3 \mathrm{~Hz}$, 1H), 7.39 (s, 1H), 7.17 (d, $J=13.3 \mathrm{~Hz}, 2 \mathrm{H}), 6.99$ (d, $J=$ $7.6 \mathrm{~Hz}, 1 \mathrm{H}), 5.60$ (s, 1H), $3.88(\mathrm{~s}, 6 \mathrm{H}), 2.38$ (s, 3H), 2.06 $(\mathrm{s}, 3 \mathrm{H}) ;{ }^{13} \mathrm{C} \mathrm{NMR}\left(75 \mathrm{MHz}, \mathrm{CDCl}_{3}\right) \delta: 172.11,157.99$, $151.72,149.27,147.59,144.47,140.15,136.24,131.88$, $128.14,127.81,126.24,120.61,118.88,82.01,53.69$, 20.98, 18.81; HRMS (ESI) calcd for $\mathrm{C}_{20} \mathrm{H}_{21} \mathrm{ClN}_{5} \mathrm{O}_{2}(\mathrm{M}+$ H) ${ }^{+}$398.1378, found 398.1376 .

2-(2'-((4"-甲基-苯基)(3"-氯-吡啶-4"-基)-亚甲基)肼基 )-4,6-二甲氧基嘧啶(III-16): 收率 93\%. m.p. 200.7 201.2 ${ }^{\circ} \mathrm{C} ;{ }^{1} \mathrm{H}$ NMR (300 MHz, DMSO) $\delta: 8.44 \sim$ $8.27(\mathrm{~m}, 2 \mathrm{H}), 7.61 \sim 7.55(\mathrm{~m}, 1 \mathrm{H}), 7.51 \sim 7.36(\mathrm{~m}, 3 \mathrm{H})$, $7.31 \sim 7.12(\mathrm{~m}, 2 \mathrm{H}), 5.61(\mathrm{~d}, J=6.3 \mathrm{~Hz}, 1 \mathrm{H}), 3.90(\mathrm{~s}, 6 \mathrm{H})$, $2.44(\mathrm{~s}, 3 \mathrm{H}) ;{ }^{13} \mathrm{C} \mathrm{NMR}\left(75 \mathrm{MHz}, \mathrm{CDCl}_{3}\right) \delta: 172.08$, 157.99 , 157.85, 151.71, 151.58, 149.28, 149.13, 147.96, $147.39,144.30,142.64,140.11,136.24,130.48,130.26$, $129.78,128.25,128.08,126.79,121.11,120.90,119.25$, 119.05, 82.49, 82.10, 53.72, 53.67, 21.12; HRMS (ESI) calcd for $\mathrm{C}_{19} \mathrm{H}_{19} \mathrm{ClN}_{5} \mathrm{O}_{2}(\mathrm{M}+\mathrm{H})^{+}$384.1222, found 384.1221 .
2-(2'-((3,4"-二甲基-苯基)(3"-氯-吡啶-4"-基)-亚甲 基)-肼基)-4,6-二甲氧基嘧啶(III-17)：收率 78\%. m.p. $171.7 \sim 171.9{ }^{\circ} \mathrm{C} ;{ }^{1} \mathrm{H}$ NMR $\left(300 \mathrm{MHz}, \mathrm{CDCl}_{3}\right) \delta: 8.44(\mathrm{~s}$, $1 \mathrm{H}), 8.29$ (d, $J=5.3 \mathrm{~Hz}, 1 \mathrm{H}), 7.49$ (dd, $J=5.3,1.5 \mathrm{~Hz}$, 1H), 7.42 (d, $J=0.9 \mathrm{~Hz}, 1 \mathrm{H}), 7.31$ (d, $J=8.2 \mathrm{~Hz}, 1 \mathrm{H}), 6.98$ (d, $J=6.3 \mathrm{~Hz}, 2 \mathrm{H}), 5.59$ (s, 1H), 3.89 (s, 6H), 2.32 (d, $J=$ $6.8 \mathrm{~Hz}, 6 \mathrm{H}) ;{ }^{13} \mathrm{C} \mathrm{NMR}\left(75 \mathrm{MHz}, \mathrm{CDCl}_{3}\right) \delta: 172.07$, $158.01,151.55,149.10,148.06,144.58,138.75,138.41$, 130.89 , 128.91, 127.26, 125.51, 121.15, 119.27, 81.96, 53.68, 19.57, 19.42; HRMS (ESI) calcd for $\mathrm{C}_{20} \mathrm{H}_{21} \mathrm{ClN}_{5} \mathrm{O}_{2}$ $(\mathrm{M}+\mathrm{H})^{+}$398.1378, found 398.1379 .

2-(2'-((4"-溴-苯基)(3"-氯-吡啶-4"-基)-亚甲基)-肼 基)-4,6-二甲氧基嘧啶(III-18)：收率 72\%. m.p. 206.8 $207.4{ }^{\circ} \mathrm{C} ;{ }^{1} \mathrm{H}$ NMR (300 MHz, $\left.\mathrm{CDCl}_{3}\right) \delta: 8.57$ (d, $J=4.9$ $\mathrm{Hz}, 0.5 \mathrm{H}), 8.29 \sim 8.20(\mathrm{~m}, 1 \mathrm{H}), 7.93(\mathrm{~s}, 0.5 \mathrm{H}), 7.69$ (d, $J=$ $8.2 \mathrm{~Hz}, 1 \mathrm{H}), 7.48 \sim 7.30(\mathrm{~m}, 3 \mathrm{H}), 7.28 \sim 7.10(\mathrm{~m}, 2 \mathrm{H}), 5.56$ $(\mathrm{d}, J=6.7 \mathrm{~Hz}, 1 \mathrm{H}), 3.84(\mathrm{~d}, J=2.1 \mathrm{~Hz}, 6 \mathrm{H}) ;{ }^{13} \mathrm{C} \mathrm{NMR}(75$ $\left.\mathrm{MHz}, \mathrm{CDCl}_{3}\right) \delta: 172.12,158.09,157.85,153.17,151.74$, $151.23,149.30,147.31,142.86,142.67,142.38,134.57$, $133.26,131.38,130.01,129.88,128.73,128.26,127.82$, $124.51,123.79,123.56,121.80,120.94,119.05,82.49$, 82.15, 53.77, 53.74; HRMS (ESI) calcd for $\mathrm{C}_{18} \mathrm{H}_{16} \mathrm{BrCl}-$ $\mathrm{N}_{5} \mathrm{O}_{2}(\mathrm{M}+\mathrm{H})^{+}$448.0170, found 448.0169.

2-(2'-((4"-苯基-苯基)(3"-氯-吡啶-4"-基)-亚甲基)肼基)-4,6-二甲氧基嘧啶 (III-19): 收率 91\%. m.p. $188.8 \sim 190.4{ }^{\circ} \mathrm{C} ;{ }^{1} \mathrm{H}$ NMR $\left(300 \mathrm{MHz}, \mathrm{CDCl}_{3}\right) \delta 8.51(\mathrm{~s}$, $1 \mathrm{H}), 8.37(\mathrm{~d}, J=5.3 \mathrm{~Hz}, 1 \mathrm{H}), 7.86(\mathrm{~d}, J=8.2 \mathrm{~Hz}, 2 \mathrm{H}), 7.72$ - $7.68(\mathrm{~m}, 2 \mathrm{H}), 7.55$ (dt, $J=14.3,4.5 \mathrm{~Hz}, 4 \mathrm{H}), 7.48-7.44$ (m, 1H), 7.40 (d, J=8.3 Hz, 2H), 5.67 (s, 1H), 3.95 (s, $6 \mathrm{H}) .{ }^{13} \mathrm{C}$ NMR $\left(75 \mathrm{MHz}, \mathrm{CDCl}_{3}\right) \delta: 172.15,158.00,151.69$, $149.26,147.81,143.90,142.84,139.38,128.76,128.75$, $128.59,128.54,128.46,127.86,126.88,126.83,126.65$, 121.14, 119.31, 82.20, 53.77; HRMS (ESI) calcd for $\mathrm{C}_{24} \mathrm{H}_{21} \mathrm{ClN}_{5} \mathrm{O}_{2}(\mathrm{M}+\mathrm{H})^{+}$446.1378, found 446.1380.

2-(2'-((3"-硝基-4"-甲基-苯基)(3"-氯-吡啶-4"-基)-亚 甲基)-肼基)-4,6-二甲氧基嘧啶(III-20)：收率 68\%. m.p. $197.2 \sim 198{ }^{\circ} \mathrm{C} ;{ }^{1} \mathrm{H}$ NMR (300 MHz, $\left.\mathrm{CDCl}_{3}\right) \delta: 8.38(\mathrm{~d}$, $J=8.7 \mathrm{~Hz}, 1 \mathrm{H}), 8.31(\mathrm{dd}, J=5.2,3.2 \mathrm{~Hz}, 1 \mathrm{H}), 7.90$ (dd, $J=15.9,1.8 \mathrm{~Hz}, 1 \mathrm{H}), 7.81(\mathrm{~d}, J=8.2 \mathrm{~Hz}, 1 \mathrm{H}), 7.61(\mathrm{~d}, J=$ $7.9 \mathrm{~Hz}, 1 \mathrm{H}), 7.54 \sim 7.44(\mathrm{~m}, 1 \mathrm{H}), 7.44 \sim 7.37(\mathrm{~m}, 1 \mathrm{H}), 7.36$ (d, $J=0.9 \mathrm{~Hz}, 1 \mathrm{H}), 5.64$ (d, $J=4.4 \mathrm{~Hz}, 1 \mathrm{H}), 3.89$ (m, 7.5H), $2.69(\mathrm{~s}, 1.5 \mathrm{H}) ;{ }^{13} \mathrm{C} \mathrm{NMR}\left(75 \mathrm{MHz}, \mathrm{CDCl}_{3}\right) \delta$ : 172.09 , 157.75, 157.66, 151.91, 151.80, 149.91, 149.52, $149.40,146.94,146.51,141.08,139.70,135.69,134.63$, $133.74,133.27,132.80,130.03,129.05,125.76,124.64$, 
120.81, 120.66, 118.96, 118.83, 82.93, 82.62, 53.86, 53.83, 20.1; HRMS (ESI) calcd for $\mathrm{C}_{19} \mathrm{H}_{18} \mathrm{ClN}_{6} \mathrm{O}_{4}(\mathrm{M}+\mathrm{H})$ 429.1073, found 429.1072.

2-(2'-((4"-叔丁基-苯基)(3"-氯-吡啶-4"-基)-亚甲 基)-肼基)-4,6-二甲氧基嘧啶(III-21)：收率 88\%. m.p. $199.1 \sim 200.7{ }^{\circ} \mathrm{C} ;{ }^{1} \mathrm{H}$ NMR (300 MHz, $\left.\mathrm{CDCl}_{3}\right) \delta: 8.42$ (s, $1 \mathrm{H}), 8.34 \sim 8.27(\mathrm{~m}, 1 \mathrm{H}), 7.64 \sim 7.57(\mathrm{~m}, 2 \mathrm{H}), 7.52 \sim 7.43$ $(\mathrm{m}, 2 \mathrm{H}), 7.23 \sim 7.17(\mathrm{~m}, 2 \mathrm{H}), 5.62(\mathrm{~s}, 1 \mathrm{H}), 3.92(\mathrm{~s}, 6 \mathrm{H})$, $1.39(\mathrm{~s}, 9 \mathrm{H}) ;{ }^{13} \mathrm{C} \mathrm{NMR}\left(75 \mathrm{MHz}, \mathrm{CDCl}_{3}\right) \delta: 172.20$, $158.04,153.21,151.57,149.16,148.03,144.36,127.95$, 126.77, 126.74, 121.15, 119.36, 81.80, 53.77, 34.65, 30.88; HRMS (ESI) calcd for $\mathrm{C}_{22} \mathrm{H}_{25} \mathrm{ClN}_{5} \mathrm{O}_{2}(\mathrm{M}+\mathrm{H})^{+} 426.1691$, found 426.1693 .

2-(2'-((4"-甲基-苯基)(4"-氯-吡啶-3"-基)-亚甲基)肼基)-4,6-二甲氧基嘧啶(III-22)：收率 64\%. m.p. 191.2 $192{ }^{\circ} \mathrm{C} ;{ }^{1} \mathrm{H}$ NMR $\left(300 \mathrm{MHz}, \mathrm{CDCl}_{3}\right) \delta: 8.50$ (d, $J=2.3 \mathrm{~Hz}, 1 \mathrm{H}), 8.36(\mathrm{~s}, 1 \mathrm{H}), 7.98(\mathrm{dd}, J=8.4,2.5 \mathrm{~Hz}$, $1 \mathrm{H}), 7.38(\mathrm{~d}, J=7.9 \mathrm{~Hz}, 2 \mathrm{H}), 7.30 \sim 7.24(\mathrm{~m}, 1 \mathrm{H}), 7.19$ (d, $J=8.0 \mathrm{~Hz}, 2 \mathrm{H}), 5.58(\mathrm{~s}, 1 \mathrm{H}), 3.89$ (s, 6H), 2.44 (s, 3H); ${ }^{13} \mathrm{C}$ NMR $\left(75 \mathrm{MHz}, \mathrm{CDCl}_{3}\right) \delta: 172.09,158.26,150.86$, 148.12 , 144.55, 139.98, 136.29, 132.48, 130.41, 128.05, 127.22, 123.39, 81.57, 53.61, 21.10; HRMS (ESI) calcd for $\mathrm{C}_{19} \mathrm{H}_{19} \mathrm{ClN}_{5} \mathrm{O}_{2}(\mathrm{M}+\mathrm{H})^{+}$384.1222, found 384.1221.

2-(2'-((2,4"-二甲基-苯基)(4"-氯一吡啶-3"-基)-亚甲 基)-肼基)-4,6-二甲氧基嘧啶(III-23)：收率 65\%. m.p. 189.4 190.8 ${ }^{\circ} \mathrm{C} ;{ }^{1} \mathrm{H}$ NMR (300 MHz, $\mathrm{CDCl}_{3}$ ) $\delta: 8.42$ (d, $J=2.0 \mathrm{~Hz}, 1 \mathrm{H}), 8.11(\mathrm{~s}, 1 \mathrm{H}), 7.93(\mathrm{dd}, J=8.4,2.5 \mathrm{~Hz}$, $1 \mathrm{H}), 7.23(\mathrm{dd}, J=8.4,0.5 \mathrm{~Hz}, 1 \mathrm{H}), 7.18 \sim 7.10(\mathrm{~m}, 2 \mathrm{H})$, $6.99(\mathrm{~d}, J=7.7 \mathrm{~Hz}, 1 \mathrm{H}), 5.54(\mathrm{~s}, 1 \mathrm{H}), 3.85(\mathrm{~s}, 6 \mathrm{H}), 2.35$ (s, 3H), 2.05 (s, 3H); ${ }^{13} \mathrm{C}$ NMR (75 MHz, $\left.\mathrm{CDCl}_{3}\right) \delta: 172.12$, $158.25,150.87,147.73,144.64,139.99,136.11,135.80$, $131.95,131.83,128.07,127.77,126.64,123.53,81.48$, 53.64, 53.57, 20.97, 18.85; HRMS (ESI) calcd for $\mathrm{C}_{20} \mathrm{H}_{21} \mathrm{ClN}_{5} \mathrm{O}_{2}(\mathrm{M}+\mathrm{H})^{+}$398.1378, found 398.1380.

2-(2'-((4"-溴-苯基)(4"-氯-吡啶-3"-基)-亚甲基)-肼 基)-4,6-二甲氧基嘧啶(III-24)：收率 89\%. m.p. 177.7 $178.5{ }^{\circ} \mathrm{C} ;{ }^{1} \mathrm{H} \mathrm{NMR}\left(300 \mathrm{MHz}, \mathrm{CDCl}_{3}\right) \delta: 8.46(\mathrm{dd}, J=2.5$, $0.5 \mathrm{~Hz}, 1 \mathrm{H}), 8.24(\mathrm{~s}, 1 \mathrm{H}), 7.94(\mathrm{dd}, J=8.4,2.5 \mathrm{~Hz}, 1 \mathrm{H})$, $7.73(\mathrm{~d}, J=8.4 \mathrm{~Hz}, 2 \mathrm{H}), 7.31 \sim 7.24(\mathrm{~m}, 1 \mathrm{H}), 7.23 \sim 7.16$ $(\mathrm{m}, 2 \mathrm{H}), 5.60(\mathrm{~s}, 1 \mathrm{H}), 3.89(\mathrm{~s}, 7 \mathrm{H}) ;{ }^{13} \mathrm{C} \mathrm{NMR}(75 \mathrm{MHz}$, $\left.\mathrm{CDCl}_{3}\right) \delta: 172.08,158.09,151.18,147.90,142.93,136.09$, $133.17,131.91,131.32,129.94,129.14,127.91,124.35$, 123.54, 81.99, 53.71; HRMS (ESI) calcd for $\mathrm{C}_{18} \mathrm{H}_{16} \mathrm{BrClN}_{5} \mathrm{O}_{2}(\mathrm{M}+\mathrm{H})^{+}$448.0170, found 448.0172.

2-(2'-((4"-(对溴苯基)-苯基)(4"-氯-吡啶-3"-基)-亚
甲基)-肼基)-4,6-二甲氧基嘧啶(III-25)：收率 66\%. m.p. 230.4 231.2 ${ }^{\circ} \mathrm{C} ;{ }^{1} \mathrm{H}$ NMR (300 MHz, $\left.\mathrm{CDCl}_{3}\right) \delta: 8.43(\mathrm{~d}$, $J=2.3 \mathrm{~Hz}, 1 \mathrm{H}), 8.10(\mathrm{~s}, 1 \mathrm{H}), 7.69(\mathrm{dd}, J=8.2,2.3 \mathrm{~Hz}$, $1 \mathrm{H}), 7.66 \sim 7.51(\mathrm{~m}, 7 \mathrm{H}), 7.45(\mathrm{~d}, J=8.6 \mathrm{~Hz}, 2 \mathrm{H}), 5.63(\mathrm{~s}$, $1 \mathrm{H}), 3.93(\mathrm{~s}, 6 \mathrm{H}) ;{ }^{13} \mathrm{C} \mathrm{NMR}\left(75 \mathrm{MHz}, \mathrm{CDCl}_{3}\right) \delta: 172.09$, $158.20,152.69,149.51,143.04,140.45,139.28,138.78$, $135.94,131.65,128.22,127.06,126.72,126.57,125.28$, 121.67, 81.97, 53.75; HRMS (ESI) calcd for $\mathrm{C}_{24} \mathrm{H}_{20} \mathrm{Br}-$ $\mathrm{ClN}_{5} \mathrm{O}_{2}(\mathrm{M}+\mathrm{H})^{+}$524.0483, found 524.0484.

2-(2'-((3,5"-二氯-苯基)(4"-氯一吡啶-3"-基)-亚甲 基)-肼基)-4,6-二甲氧基嘧啶(III-26), 收率 61\%. m.p. $192.4 \sim 193.8{ }^{\circ} \mathrm{C} ;{ }^{1} \mathrm{H}$ NMR $\left(300 \mathrm{MHz}, \mathrm{CDCl}_{3}\right) \delta: 8.44 \sim$ $8.39(\mathrm{~m}, 1 \mathrm{H}), 8.10$ (s, 1H), $7.94(\mathrm{dd}, J=8.4,2.5 \mathrm{~Hz}, 1 \mathrm{H})$, $7.63(\mathrm{~d}, J=1.9 \mathrm{~Hz}, 1 \mathrm{H}), 7.48$ (dd, $J=8.2,2.0 \mathrm{~Hz}, 1 \mathrm{H})$, 7.30 (dd, $J=8.4,0.6 \mathrm{~Hz}, 1 \mathrm{H}), 7.23$ (d, $J=8.2 \mathrm{~Hz}, 1 \mathrm{H})$, $5.62(\mathrm{~s}, 1 \mathrm{H}), 3.89(\mathrm{~d}, J=10.2 \mathrm{~Hz}, 6 \mathrm{H}) ;{ }^{13} \mathrm{C} \mathrm{NMR}(75 \mathrm{MHz}$, $\left.\mathrm{CDCl}_{3}\right) \delta: 172.10,157.98,151.22,147.38,140.06,137.06$, $135.62,134.08,130.98,130.69,128.57,127.84,123.72$, 82.13, 53.78; HRMS (ESI) calcd for $\mathrm{C}_{18} \mathrm{H}_{15} \mathrm{Cl}_{3} \mathrm{~N}_{5} \mathrm{O}_{2}(\mathrm{M}+$ $\mathrm{H})^{+} 438.0286$, found 438.0288 .

辅助材料(Supporting Information) 26 个芳基吡啶酮 腙类化合物对番茄晚疫病菌初篎图片、化合物 III-3 和 III-14 对番茄晚疫病菌精密毒力抑菌图片. 这些材料可 以免费从本刊网站(http://sioc-journal.cn/)上下载.

\section{References}

[1] Ameen, D.; Snape, T. J. Med. Chem. Commun. 2013, 4, 893.

[2] Liu, X. L.; Xiao, Y. M.; Li, J. Q.; Fu, B.; Qin, Z. H. Mol. Diversity DOI: 10.1007/S11030-018-9895-3.

[3] Song, M. Y.; Cao, C. Y.; He, Q. R.; Dong, Q. M.; Li, D.; Tang, J. J.; Gao, J. M. Bioorg. Med. Chem. 2017, 25, 5290.

[4] Roszkowski, A. P. J. Pharmacol. Exp. Ther. 1965, 149, 288.

[5] Abrahamsson, S.; Nilsson, B. J. Org. Chem. 1966, 31, 3631.

[6] Anon Fain Kemikaru 2010, 39, 39.

[7] Krol, M.; Podsadni, P.; Turlo, J.; Ufnal, M.; Drapala, A.; Szulczyk, B.; Dawidowski, M. Eur. J. Pharm. Sci. 2016, 81, 42.

[8] Reder R. F.; Danilo P. J.; Rosen M. R. Eur. J. Pharmacol. 1980, 61, 321.

[9] Hart, T.; Macias, A. T.; Benwell, K.; Brooks, T.; D'Alessandro, J.; Dokurno, P.; Francis, G.; Gibbons, B.; Haymes, T.; Kennett, G.; Lightowler, S.; Mansell, H.; Matassova, N.; Misra, A.; Padfield, A.; Parsons, R.; Pratt, R.; Robertson, A.; Walls, S.; Wong, M.; Roughley, S. Bioorg. Med. Chem. Lett. 2009, 19, 4241.

[10] Tanaka, I.; Otani, S.; Nakayashiki, J.; Fujita, S. Yakuzaigaku 1964, 24,303 .

[11] Tanaka, I.; Otani, S.; Seki, T.; Shinkai, H.; Kurashige, T. Yakuzaigaku 1965, 25, 31.

[12] Che, C. L.; Yang, D. Y.; Wan, C.; Wang, J. Y.; Liu, X. L.; Zhao, F. H.; Qin, Z. H. Chin. J. Pestic. Sci. 2017, 19, 533 (in Chinese). (车传亮, 杨冬燕, 万川, 王家尧, 刘雪莲, 赵峰海, 覃兆海, 农 药学学报, 2017, 19, 533.)

[13] Mu, C. W.; Yuan, H. Z.; Li, N.; Fun, B.; Xiao, Y. M.; Ma, Y. Q.; Qi, S. H.; Qin, Z. H. Chem. J. Chin. Univ. 2007, 28, 1902 (in Chinese) (慕长炜, 袁会珠, 李楠, 傅滨, 肖玉梅, 马永强, 齐淑华, 覃兆 
海, 高等学校化学学报, 2007, 28, 1902.)

[14] Xiao, Y. M.; Esser, L.; Zhou, F.; Li, C.; Zhou, Y. H.; Yu, C.-A.; Qin, Z. H.; Xia, D. PLoS One 2014, 9, e93765.

[15] Yan, X. J.; Qin, W. C.; Sun, L. P.; Qi, S. H.; Yang, D. B.; Qin, Z. H.; Yuan, H. Z. J. Agric. Food Chem. 2010, 58, 2720.

[16] Xiao, Y. M.; Yang, X. L.; Li, B.; Yuan, H. Z.; Wan, S. Q.; Xu, Y. J.; Qin, Z. H. Molecules 2011, 16, 8945.

[17] Li, X.; Han, X.; He, M. M. Xiao, Y. M.; Qin, Z. H. Bioorg. Med. Chem. Lett. 2016, 26, 5936.

[18] Li, X. Y.; Wan, C.; Du, S. J.; Li, H.; Yuan, H. Z.; Jiang, J. Z.; Xiao, Y. M.; Qin, Z. H. Chem. J. Chin. Univ. 2015, 36, 2415 (in Chinese). (李秀云, 万川, 杜士杰, 李红, 袁会珠, 蒋家珍, 肖玉梅, 覃兆 海, 高等学校化学学报, 2015, 36, 2415.)

[19] Zhang, L. M.S. Thesis, China Agricultural University, Beijing, 2017 (in Chinese).
(张否, 硕士学位论文，中国农业大学，北京，2017.)

[20] Okuno, T.; Furusawa, I.; Matsuura, K.; Shishiyama, J. A. Phytopathol. Soc. Jpn. 1989, 55, 281.

[21] Cechin, S. R.; Schetinger, M. R. C.; Zanatta, N.; Madruga, C. C.; Pacholski, I. L.; Flores, D. C.; Bonacorso, H. G.; Martins, M. A. P.; Morsch, V. M. Chem. Res. Toxicol. 2003, 16, 1433.

[22] Bonnett, S. A.; Ollinger, J.; Chandrasekera, S.; Florio, S.; O'Malley, T.; Files, M.; Jee, J. A.; Ahn, J.; Casey, A.; Ovechkina, Y.; Roberts, D.; Korkegian, A.; Parish, T. ACS Infect. Dis. 2016, $2,893$.

[23] Chen, N. C. Pesticide Bioassay Technology, China Agriculture Press, Beijing, 1991, pp. 208 239 (in Chinese). (陈年春，农药生物测定技术，中国农业出版社，北京，1991，pp. 208 239.)

[24] Konishi, K.; Kuragano, T.; Matsuura, K. Agric. Biol. Chem. 1986, 50, 2427.

(Zhao, C.) 